\title{
AN EXPLANATION TO THE ANOMALOUS AVRAMI EXPONENT
}

\author{
N.X. Sun, X.D. Liu*, and K. Lu* \\ National Key Laboratory for $R S A$, *also with International Centre for \\ Materials Physics, Institute of Metal Research, Academia Sinica, \\ Shenyang 110015, P. R. China \\ (Received July 9, 1995)
}

\section{Introduction}

Quantitative description of the isothermal crystallisation kinetics of amorphous solids is often made by means of the concept first developed by Kolmogorov (1), and later independently derived by Johnson and Mehl (2) and Avrami (3). If the following two assumptions are made: (a) nucleation rate is either zero (i.e. crystallisation occurs due to the growth of pre-existing nuclei) or constant, and (b) isotropical growth rate is proportional to either time $t$ or $t^{0.5}$ (depending whether the devitrification is interface or diffusion controlled), then the classical Kolmogorov-Johnson-Mehl-Avrami (KJMA) equation can be derived to be (4):

$$
x(t)=1-\exp \left[-K(t-\tau)^{n}\right]
$$

where $x(t)$ is the volume fraction transformed at time $t, \tau$ is the incubation time or time lag, and $n$ is the Avrami exponent which reflects the nucleation rate and/or the growth morphology. $K$ is the pre-exponential factor, a kinetic parameter depending on the annealing temperature, nucleation rate and growth rate. For isothermal crystallisation, the Avrami plot of $\ln \ln (1-x)^{-1}$ versus $\ln (t-\tau)$ should give a straight line with a slope of $n$.

However, many anomalous Avrami exponents and non-linear Avrami plots have been observed in the crystallisation processes of various metallic glasses (5-11). Greer (5) has pointed out that the average Avrami exponents, $n$, for the crystallisation process of Metglas $2605 \mathrm{Fe}_{80} \mathrm{~B}_{20}$ alloys differ from 0.7 to 4.87 , but no explanation was proposed for the abnormally low values of $n$. Nicolaus et al. (6) have studied the crystallisation kinetics of amorphous $\mathrm{Co}_{33} \mathrm{Zr}_{67}$ alloys, they found out that the average Avrami exponents were not consistent at different temperatures, and they obtained a mean Avrami exponent of $n=2.44$, which is also lower than 3.0, the expected value for a polymorphic crystallisation process according to the KJMA equation, and cannot be explained by the classical KJMA equation.

Schaafsma et al. (7) have observed a decrease in local Avrami exponents $n(x)$ (defined as $n(x)=\partial \ln l n$ $\left.(1-x)^{-1 / \partial}(t-\tau)[8]\right)$ at high $x$ in the crystallisation process of Metglas $2605 \mathrm{Fe}_{80} \mathrm{~B}_{20}$ alloy, which they attributed to the delayed crystallisation near the substrate side of the ribbons obtained by melt-spinning.

However, Ghosh et al. (9) have observed an abnormal rise of the local Avrami exponents at high $x$ in the crystallisation process of amorphous $\mathrm{Ni}_{24} \mathrm{Zr}_{76}$ alloy ribbons; $\mathrm{Lu}$ et al. (10) have detected a similar rise of $n(x)$ in the crystallisation process of amorphous $\mathrm{Ni}_{33} \mathrm{Zr}_{67}$ alloy ribbons. This abnormal rise of $n(x)$ was also observed 


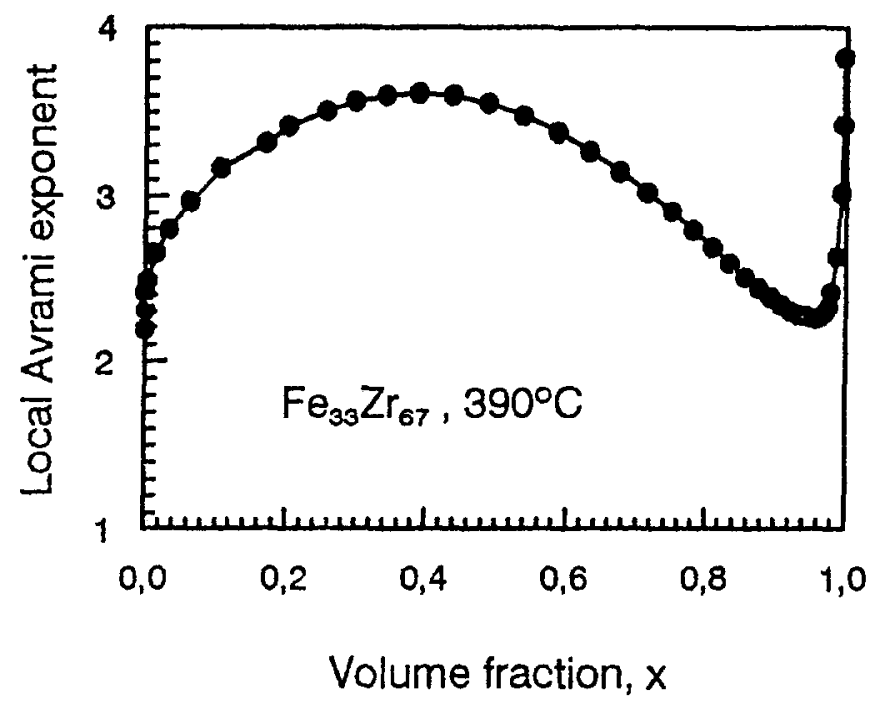

Figure 1. Local Avrami exponent, $n(x)$, $v s$, the transformed volume fraction for the crystallisation process of amorphous $\mathrm{Fe}_{33} \mathrm{Zr}_{67}$ alloy ribbons at $390^{\circ} \mathrm{C}$.

in the crystallisation process of amorphous $\mathrm{Fe}_{33} \mathrm{Zr}_{67}$ alloys, as shown in Fig. 1 (11), which is a typical plot of the anomalous local Avrami exponents: when $x>40 \%, n(x)$ drop continuously from 3.6 to a minimum of 2.3 at $x=95 \%$ and then rise sharply from below 2.3 to about 4.0 at $x=100 \%$.

Ghosh et al. (9) have proposed that the impingement effect at the final stage may account for the abnormal rise of the local Avrami exponents at high $x$, but it needs further theoretical and experimental confirmation. Holzer et al. (12) have observed that the Avrami exponents were in the range 2 2.5 for the polymorphic crystallisation process of melt-spun amorphous $\mathrm{Al}-\mathrm{Cu}-\mathrm{V}$ alloys. They claimed that, these anomalous Avrami exponents arose from an inhomogeneous distribution of pre-existing nuclei, and they derived a modified KJMA equation using a concept of "extended area fraction," but it is related with a lot of parameters and seems inconvenient.

Here, by assuming a spatial distribution of nuclei, i.e. a spatial distribution of pre-existing nuclei and/or nucleation rate, a modified form of KJMA equations is proposed, which can account for the widely observed anomalous average Avrami exponents and the abnormal drop and rise of local Avrami exponents $n(x)$ at high $x$, i.e. non-linear Avrami plots in the crystallisation process of various amorphous alloys.

\section{Analysis}

Anomaly of the (local) Avrami exponents and/or nonlinearity of the Avrami plots can be caused by various reasons such as: (i) different mechanisms controlling in the crystallisation process (13); (ii) a time dependent nucleation rate (14); (iii) possibility of simultaneous grain growth of the crystallised region during crystallisation; (iv) impingement effect, which is important especially at the final stage of crystallisation, if the anisotropy of the crystals is sufficiently high (15); and some other reasons, etc. But it seems that none of them can account for the anomaly of the (local) Avrami exponents mentioned above satisfactorily.

As Johnson and Mehl (3) have pointed out that their analysis pertains to reactions in which nuclei form at random throughout the matrix, without regard for the matrix structure, but if the pre-existing and/or newly formed nuclei distribute inhomogeneously, the Avrami exponent will be changed. 
A moditied form of KJMA equations has been derived by assuming a spatial distribution of the density of pre-existing nuclei, $n(y)$, and nucleation rate, $i(y)$, along the thickness of the ribbon, $y$. If the isotropic growth rate, $u$, is linear (as for interface controlled growth), then, the particle radius, $r$, at time $t$ is

$$
r=u t
$$

or if the growth is controlled by diffusion (volume controlled), it normally follows a parabolic law, the particle radius, $r$, is then

$$
r=A(D t)^{0.5}
$$

where $A$ is a constant of the order of magnitude one and $D$ is the diffusion coefficient. If $\mathrm{d} V^{\beta}$ is the volume of the newly formed $\beta$ phase in volume $\mathrm{d} y$, and $\mathrm{d} V_{e x}^{\beta}$ is the extended volume of the $\beta$ phase, then

$$
\mathrm{d}\left(\mathrm{d} V^{\beta}\right) /\left(\mathrm{d} y-\mathrm{d} V^{\beta}\right)=\mathrm{d}\left(\mathrm{d} V_{e x}^{\beta}\right) / \mathrm{d} y
$$

Integrating equation 4 , we get

$$
-\mathrm{d} V_{e x}^{\beta} / \mathrm{d} y=\ln \left(1-\mathrm{d} V^{\beta} / \mathrm{d} y\right)
$$

for linear growth rate, $r=u t$, so

$$
\mathrm{d} V_{e x}^{\beta}=(4 \pi / 3) \int_{0}^{t} u^{3}(t-\tau)^{3} \mathrm{~d} \tau i(y) \mathrm{d} y+(4 \pi / 3) u^{3} t^{3} n(y) \mathrm{d} y
$$

while for parabolic growth, $r=A(D t)^{0.5}$, then

$$
\mathrm{d} V_{e x}^{\beta}=(4 \pi / 3) \int_{0}^{t}\left\{A[D(t-\tau)]^{0.5}\right\}^{3} \mathrm{~d} \tau i(y) \mathrm{d} y+(4 \pi / 3)\left[A(D t)^{0.5}\right]^{3} n(y) \mathrm{d} y
$$

Substituting equations 6 and 7 into equation 5 respectively, and integrating them, we get the modified form of KJMA equations for interface controlled and diffusion controlled growth mechanisms, respectively,

(i) linear growth

$$
x(t)=V^{\beta} / h=1-(1 / h) \int_{0}^{h} \exp \left[-(\pi / 3) u^{3} t^{4} i(y)-(4 \pi / 3) u^{3} t^{3} n(y)\right] \mathrm{d} y
$$

(ii) parabolic growth

$$
x(t)=: V^{\beta} / h=1-(1 / h) \int_{0}^{h} \exp \left[-(8 \pi / 15) i(y) A^{3} D^{3} t^{5 / 2}-(4 \pi / 3) A^{3} D^{3} t^{3 / 2} n(y)\right] \mathrm{d} y
$$

where $h$ is the thickness of the ribbon, $x(t)$ is the volume fraction of the transformed $\beta$ phase.

\section{Results and Discussion}

There have been a lot of experimental studies and theoretical analyses of the flow of heat and material in melt-spinning (16-18), and there exists compositional and structural inhomogeneity along the thickness of the melt-spun ribbons $(7,12)$, but quantitative analysis of $n(y)$, the density of pre-existing nuclei, and $i(y)$, the nucleation rate, along the thickness $y$ is still lacking to our knowledge, and requires further investigation. In order to show the effects of the inhomogeneity of the nuclei distribution on the Avrami exponent, we assume that 


$$
\begin{gathered}
n(y) N_{0}+N_{1} y \\
i(y) I_{0}+I_{1} y
\end{gathered}
$$

where $N_{0}, N_{1}$ and $I_{0}, I_{1}$ are coefficients. So, $N_{1}$ and $I_{1}$ can be a quantitative description of the inhomogeneity of the nuclei distribution. Then, different Avrami exponents can be obtained by substituting equations 10 and 11 into equation 8 for varied $N_{0}, N_{1}$ and $I_{0}, I_{1}$ (because the available Avrami exponents for diffusion controlled crystallisation processes are rare, so discussion is only confined to equation 8 ).

If $N_{1}=I_{1}=0$, i.e. a random distribution of nuclei, then equation 8 will have the form of equation 1 , i.e. the classical KJMA equation. According to equation 8, the Avrami exponent $n$ is 3.0 when $I_{0}=I_{1}=N_{1}=$ 0 ; however, if $N_{1} \neq 0$, the Avrami exponent will be changed. Figure 2 shows the corresponding curves of volume fraction $x(t)$ versus time $t$, the Avrami plot $\ln \ln (1-x)^{-1} v s \ln t$, and the local Avrami exponents $n(x)$ $v s x$ with different $N_{1}$. It is evident that, with the increase of $N_{1}$ the deviation of the Avrami plot from a straight line is more and more pronounced and the anomaly of the local Avrami exponents is also more and more obvious. When $N_{1}=1.0 \times 10^{26} \mathrm{~m}^{-4}$, the Avrami plot is actually a straight line, and the corresponding local Avrami exponents $n(x)$ is nearly 3.0; however, when $N_{1}=5.0 \times 10^{28} \mathrm{~m}^{-4}$, nonlinearity of the Avrami plot is obvious at high $x$, and the corresponding $n(x)$ drop continuously from $n=3.0$ at $x$ $=0$, to a minimum of $n=1.3$ at $x-97 \%$, and then rise sharply to 2.0 at $x=100 \%$, a similar abnormality as those experimentally obtained. Also, when $N_{0}=N_{1}=I_{1}=0$, an Avrami exponent $n=4.0$ is expected in classical KJMA analysis; if $I_{1} \neq 0$, the three kinds of curves as those in Fig. 2 are also obtained and shown in Fig. 3, similar results as those in Fig. 2 have been obtained.

From Figs. 2 and 3, it is evident that the $n(x)$ is $x$ curves actually overlap at low $x$, which means that at low $x$, the local Avrami exponents are the same without regard for the distribution of the nuclei, so, the local Avrami exponents at low $x$ can reflect the nucleation rate and/or the growth morphology, just the same as the Avrami exponent of the classical KJMA equation; with the increase of $x$, the $n(x)$ curves begin to deviate from a straight line, further, with the increase of $N_{1}$ and/or $I_{1}$, the deviation is more and more obvious, that is, the more inhomogeneous distribution of nuclei, the lower the Avrami exponents; at high $x$ (for example, $x>95 \%$ ), the local Avrami exponents rise sharply from the minimum value of $n(x)$. The anomaly of Avrami exponents is also reflected in the Avrami plots.

From the discussion above, one can see that inhomogeneous distribution of nuclei will result in the following two effects:

(a) lowering the local Avrami exponents, therefore the average Avrami exponent,

(b) nonlinearity of the Avrami plot, especially at high $x$.

If there exist inhomogeneously distributed pre-existing nuclei in the ribbons, and/or a inhomogeneous nucleation occurs in the crystallisation process, anomalous Avrami exponent and non-linear Avrami plot will be expected, just as what have been observed in references (5-11). So, the inhomogeneous distribution of nuclei in the ribbon samples may be one important factor resulting in the widely observed anomalous Avrami exponents and non-linear Avrami plots.

Then, one should be careful in using the Avrami exponent to determine the nucleation rate and/or growth morphology, especially with the presence of inhomogeneous distribution of nuclei. The $n(x)$ at high $x$ and the average Avrami exponent will not reflect the nucleation rate and/or growth morphology correctly, if the nuclei distribute inhomogeneously; while the local Avrami exponents at low $x$ are actually unaffected by the inhomogeneity of the distribution of nuclei, when the inhomogeneity of the distribution of nuclei is not high enough, as can be seen in Figs. 2 and 3, so, one can still use the local Avrami exponents at low $x$ to determine the nucleation rate and/or growth morphology. 

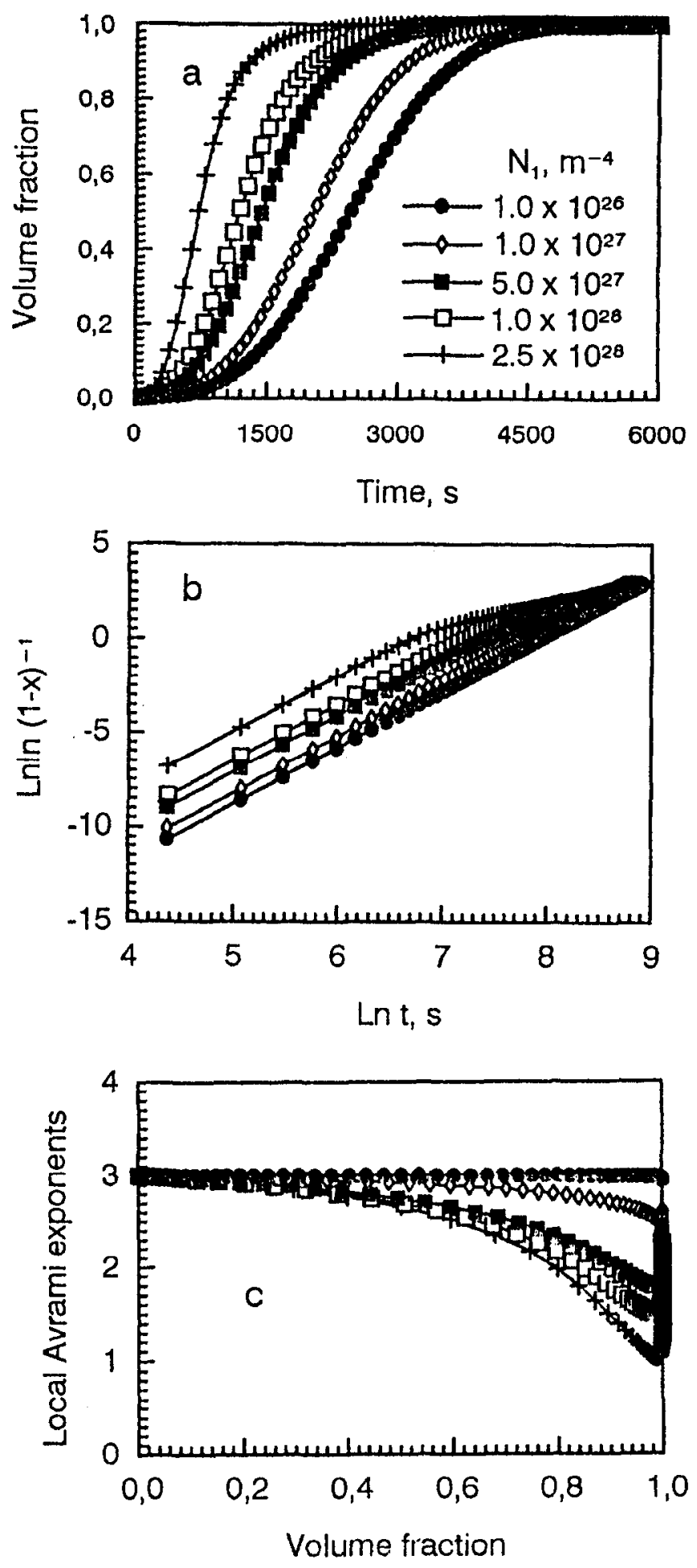

Figure 2. Simulated plots with varied $N_{\mathrm{b}}$, (a): $x(t)$ versus $t$, (b): $\ln \ln (1-x)^{-1}$ versus $\ln t$, (c): $n(x)$ versus $x$, with $I_{1}=I_{0}=0, N_{0}=1.0$ $\times 10^{22} \mathrm{~m}^{-3}, u=1.0 \times 10^{-11} \mathrm{~m} / \mathrm{s}, h=10^{-5} \mathrm{~m}$. 

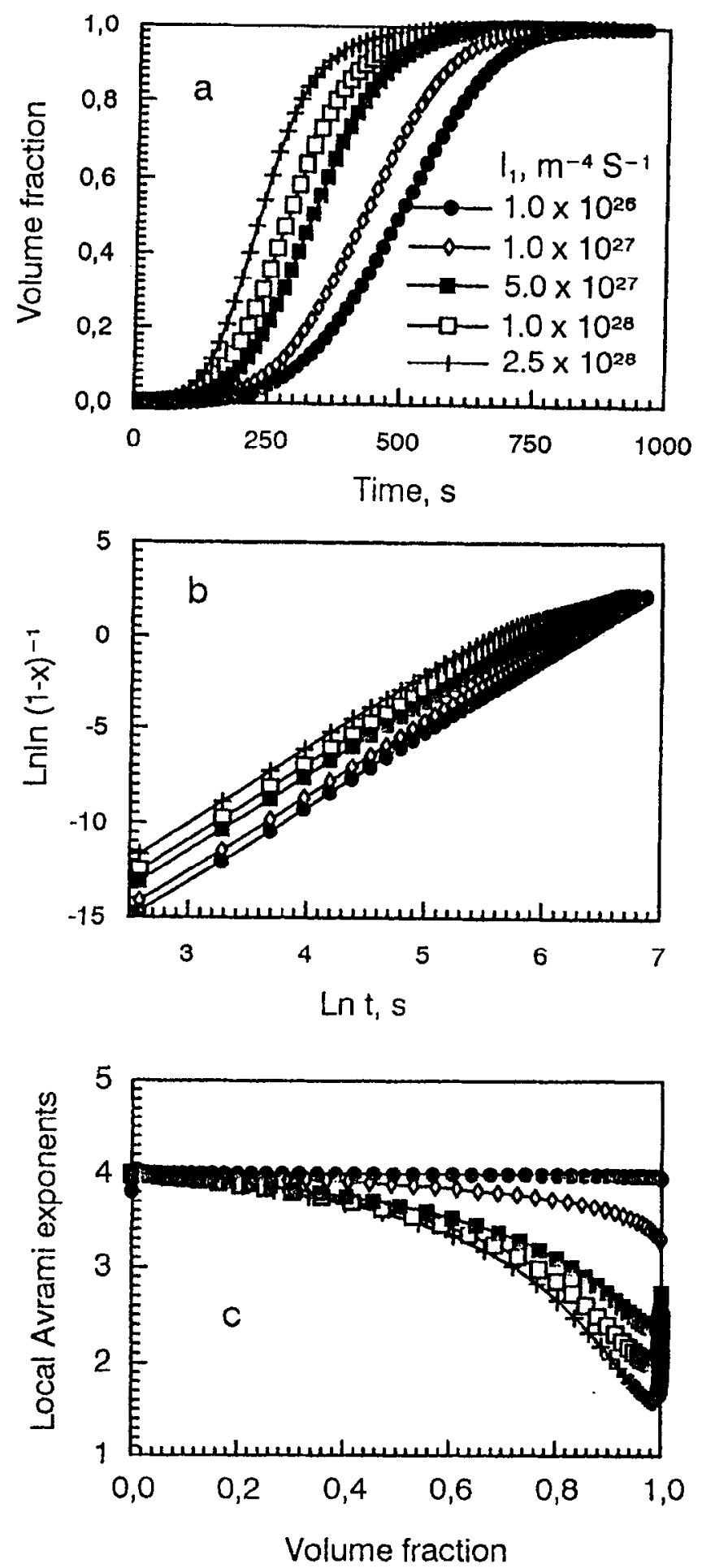

Figure 3. Simulated plots with varied $I_{1}$, (a): $x(t)$ versus $t$, (b): $\ln \ln (1-x)^{-1}$ versus $\ln t$, (c): $n(x)$ versus $x$, with $N_{1}=N_{0}=0, I_{0}=1.0$ $\times 10^{22} \mathrm{~m}^{-3}, u=1.0 \times 10^{-11} \mathrm{~m} / \mathrm{s}, h=2.0 \times 10^{-5} \mathrm{~m}$. 


\section{Summary}

A modified form of KJMA equations has been derived by assuming a distribution of pre-existing nuclei, and nucleation rate. It was found that the inhomogeneous distribution of nuclei can result in two effects:

(a) lowering the Avrami exponent,

(b) nonlinearity of the Avrami plot, especially at high $x$.

The modified form of KJMA equations can be an explanation to the widely observed anomalous Avrami exponents and nonlinear Avrami plots.

\section{Acknowledgements}

The authors thank Prof. U. Köster for helpful discussion. The financial supports from the National Natural Science Foundation of China and the Chinese Academy of Sciences are acknowledged.

\section{References}

1. A.N. Kolmogorov, Bull. Acad. Sci. U.S.S.R. Phys. Ser. 3, 555 (1937).

2. A.M. Johnson and R.F. Mehl, Trans. Am. Inst. Min. Engrs 135, 417 (1939).

3. M. Avrami, J. Chem. Phys. 7, 1103 (1939); ibid, 8, 212 (1940), ibid, 9, 177 (1941).

4. J.W. Christian, The Theory of Transformations in Metals and Alloys, 2nd edn. Pergamon Press, Oxford (1975).

5. A.L. Greer, Acta Metall. 30,171 (1982).

6. M.M. Nicolaus, H.-R. Sinning, and F. Hassner, Mat. Sci. Eng. Al50, 101 (1992).

7. A.S. Schaafsma, H. Snijders, F. van der Woude, J. w. Drijver and S. Radelaar, Phys. Rev. B. 20,4423 (1979).

8. A. Calka and A.P. Radlinski, MRS Symp. Proc. 80, 203 (1987).

9. G. Ghosh, M. Chandrasekaran and L. Delaey, Acta Metall. 39, 925 (1991).

10. K. Lu, X.D. Liu and F.H. Yuan, Physica B, 1995, in press.

11. N.X. Sun, X.D. Liu and K. Lu, to be submitted to Nanostruct. Mater.

12. J.C. Holzer and K.F. Kelton, Acta Metall. 39, 1833 (1991).

13. R. Luck, K. Lu and W. Frantz, Scripta Metall. 28, 1071 (1993).

14. Z. Altounian, C.A. Volkert and J.O. Ström-Olson, J. AppI. Phys. 57, 1777 (1985).

15. M.P. Shepilov and D.S. Baik, J. Non-cryst. Solids. 171, 141 (1994).

16. T.R. Anthony and H.E. Cline, J. Appl. Phys. 49, 829 (1978).

17. H. Hillmann and H.R. Hilzinger, Rapidly Quenched Metals III (edited by B. Cantor), Vol. 1. p. 28. Metals Society, London (1978).

18. E.M. Gutierre: and J. Szekely, Metall. Trans. B, 17, 695 (1986). 